\title{
Time-resolved measurement of shear-band temperature during serrated flow in a Zr-based metallic glass
}

\author{
P. Thurnheer, F. Haag, J. F. Löffler \\ Laboratory of Metal Physics and Technology, Department of Materials, ETH Zurich, 8093 Zurich, Switzerland
}

\begin{abstract}
Severe localization of plastic flow into shear bands is typically accompanied by local heating. In metallic glasses, the small lateral extension of shear bands, the short duration of their intermittent plastic slips, and the unpredictable position of their initiation make in-situ investigations of local changes in temperature during shear banding difficult. An alternative is to estimate shear-band temperature using calculations for a given assumed deformation model, which generates predictions of temperature changes from $5^{\circ} \mathrm{C}$ to more than $1000^{\circ} \mathrm{C}$. In this study we review four models of plastic slip accumulation and present first results on temporally resolved shear-band heating in metallic glasses via infrared thermometry. We find that the heat evolution in a shear band during plastic slip is best modelled by shear that occurs simultaneously within serration time scales. This illustrates clearly that temperature changes within shear bands are less than $6^{\circ} \mathrm{C}$ during stable plastic flow.
\end{abstract}

Keywords: Metallic glasses, IR thermography, Serrated flow, Shear bands, Mechanical properties

${ }^{*}$ Corresponding author.

E-mail address: joerg.loeffler@mat.ethz.ch (J.F. Löffler). 


\section{Introduction}

At temperatures well below their glass transition, the plastic deformation of metallic glasses is manifested by shear steps on the sample surface. These steps are caused by plastic flow, which is localized within narrow regions of weakened material called shear bands [1-6]. Because shear bands are as thin as $10 \mathrm{~nm}$ [7], initiate at locations which are difficult to predict, and operate intermittently for short durations only $[8,9]$, in-situ investigation of the accumulation of plastic strain in metallic glasses (MGs) is a challenging task. It is thus not surprising that since the first reports on the mechanical behaviour of MGs [10], different perceptions have emerged regarding the exact mechanism by which shear steps are generated. This is not trivial, because the choice of a specific model has immediate consequences for the estimate of shear-band properties that are not directly measured experimentally, such as the speed at which the shear step is generated (the shear-band velocity $v_{\mathrm{SB}}$ ), the shear-band viscosity $\eta$ and the temperature rise during shear-band operation $\Delta T$. The latter parameter is of particular interest, since a critical temperature jump is the core element in at least two theoretical studies attempting to understand size- and compliance-induced MG embrittlement [11, 12]. While both of these studies state that the degradation of mechanical properties is caused by shearband temperature exceeding a critical value, they disagree on how a shear step is generated, and thus derive different temperatures within a shear band. Such unconformities are wide-spread in literature and indicate the necessity of the present work.

The aim of this work is twofold: (1) to give an overview of the different perceptions regarding shear-step generation found in literature, and (2) to test their agreement with new experimental results regarding time-resolved shear-band heating during inhomogeneous MG flow. Both (1) and (2) will contribute to a detailed understanding of the shear-step generation mechanism in deforming MGs.

\section{Models of shear-step generation during inhomogeneous plastic flow}

Fig. 1a illustrates the four most important models of shear-step generation. These models are labelled as (1) phonon-shear-front propagation, (2) serration-shear-front propagation, (3) serrationtransient behaviour, and (4) serration-simultaneous shear. 
A first means of distinguishing between these models is their approach to flow curves obtained by instrumented mechanical testing. In these curves, plastic flow is characterized by stick-slip discontinuities called serrations. A sequence of three serrations is schematically depicted in Fig. 1b, where each consists of a slow loading phase (stick, light grey) and a fast unloading phase (slip, darker grey). The slip phases appear simultaneously in the load and displacement signal, and are characterized by the size of the load drop $\Delta F$, the apparent displacement jump $\Delta u_{0}$, and the serration duration $\Delta t$. For better illustration, these serration parameters are taken as positive quantities. While models (2)-(4) assume that the serration parameters correctly reflect the increment size of the shear step and the duration over which it is established, the phonon-shear-front propagation model (1) does not take such serrations into account.

\subsection{Phonon-shear-front propagation model}

Georgarakis et al. [13] followed the approach that if the shear-step increment was calculated from each serration, then the sum of all these increments should add up to the total plastic strain. Finding that the sum of apparent displacement jumps $\Delta u_{0}$ strongly underestimates the true total size of the shear steps, the authors inferred that the time scales must also be compromised, and concluded that the stress serrations measured by instrumented mechanical testing are measurement artefacts rather than a direct probe of shear-step size and shear-band dynamics. Acknowledging that a shear band cannot be formed instantaneously, they attributed both the generation of the shear band and the formation of the shear step to the propagation of a dilating shear wave that travels through the sample at a fraction of the speed of sound. When taking into account the presence of a stress-redistribution field around the shear front, the time for the generation of a shear step of $1 \mu \mathrm{m}$ size then accounts to $10^{-8} \mathrm{~s}$. This led the authors to predict temperature rises of thousands of ${ }^{\circ} \mathrm{C}$ within the shear band [13]. Such high values would explain reports of the establishment of a micron-sized heat-affected zone around the shear band [14] and of shear banding which causes the melting of fusible tin coatings on top of an emerging shear step [15]. This scenario is an integral part of the model of Miracle et al. regarding the size effect in MG ductility [12]. However, a recent study [16] has revealed that melting of a tin coating is not universally observed in MG deformation, but is rather a function of alloy and stress state. This eliminates the condition that shear bands must "necessarily" be hot. 


\subsection{Models (2), (3) and (4), using serration time scales}

In contrast to this phonon-based model, various studies report a direct correlation between shear steps and the features of serrated flow curves from instrumented mechanical testing [17, 18] (Fig. 1a, (2)-(4)). These models assume that the time scale for a single serration corresponds to the time scale of the coincident shear-step formation, and that the shear step of a given amplitude $\Delta u_{\mathrm{pl}}$ can be calculated via [19]

\section{Equation 1}

$$
\Delta u_{\mathrm{pl}}=\sqrt{2}\left(\Delta u_{0}+\Delta F C_{\mathrm{S}}\right)
$$

According to Equation 1, the shear step consists of the apparent displacement jump $\Delta u_{0}$, measured via strain gauges, plus a term accounting for the elastic recovery of the sample during the serration, where $C_{\mathrm{S}}$ is the sample compliance. The factor $\sqrt{2}$ converts uniaxial displacement to displacement on the shear plane, which is oriented $\approx 45^{\circ}$ to the load axis. It is noted that the second term in Equation 1 was neglected by Georgarakis et al. [13] and that its omission may be the true cause of the abovementioned mismatch between the sum of flow-curve strain increments and the total shear-step sizes. The apparent displacement jump $\Delta u_{0}$ can further be related to the compliance of the testing setup $C_{\mathrm{M}}$ via $\Delta u_{0}=\Delta F C_{\mathrm{M}}$, leading us to an alternative formulation of Eq. 1 , which is $\Delta u_{\mathrm{pl}}=\sqrt{2} \Delta F\left(C_{\mathrm{M}}+C_{\mathrm{S}}\right)$ $[20]$.

Although models (2)-(4) agree on serration time scales, they differ in their understanding of how plastic strain is accumulated by a shear band. Model (2) assumes that the shear step forms via a propagating front [19]. In other words, the full shear step is established behind the shear front, while the material ahead of the front remains undeformed. In this case, the shear-band velocity, i.e. the speed at which the shear step is generated, can be calculated as the ratio of the fully established shear band across the sample of diameter $d$ and the serration time $\Delta t$, i.e. $v=\sqrt{2} d / \Delta t$. In contrast, model (4) assumes that the shear step is established simultaneously along the shear plane [21]. In that case, it is the shear step only that is divided by the event duration in the calculation of the shear-band velocity, i.e., $v=\Delta u_{\mathrm{pl}} / \Delta t$, with $\Delta u_{\mathrm{pl}}$ given by Equation 1. These calculations provide shear-band velocities that are smaller by a factor of $10^{3}$ compared to those calculated from model (2) for a sample of a few millimetres in diameter. Note, however, that the velocities calculated from model (2) are still three 
orders of magnitude smaller than those obtained from model (1). With high-speed imaging methods, Song et al. [22] and Wright et al. [23] directly observed the scenario of model (4) down to time scales of about $0.2 \mathrm{~ms}$, i.e. a simultaneous deformation on the shear plane within their temporal resolution. They also did not resolve any delayed onset of shear banding on either side of the sample. The perception of sub-resolution intermittency, a necessity for reconciling the above mentioned experimental finding with model (2), was also made unlikely by recent Acoustic Emission (AE) measurements [24], which revealed only one shear-band initiation signal per serration. From that perspective, scenario (2) may be discarded. Most recently, Joo et al. [25] carried out experiments using digital image correlation (DIC) on MGs under compression. The authors observed a transition from front-propagating behaviour of the shear band in the early stage of deformation (associated with small strain increments only) to simultaneous shear in the subsequent stage, during which the major part of the total shear step was generated [25]. This is the scenario (3) presented in Fig. 1a. Similar results regarding a transition from a propagating to a simultaneous flow mode were obtained in a recent study by Qu et al. [26]. These authors report on shear steps that form via a propagating flow mode in the early regime of "apparent strain hardening", i.e. prior to reaching peak stress, and a transition to simultaneous shearing once a major shear band has formed. In a purely strain-weakening material, such as a metallic glass, events prior to reaching peak stress cannot be system-spanning and must thus be associated with local geometric constraints. From that perspective, we may deduce that scenario (4) is an idealized case of scenario (3) and shows an almost perfect elastic-to-plastic transition, devoid of geometrically-induced artefacts that would give rise to apparent front-propagating shear banding at pre-peak plastic strains. Initial apparent propagation thus appears to be related to stress-inhomogeneities within the MG samples rather than to a deformation mechanism according to model (2) in Fig. 1a.

Despite the fact that shear steps can be derived from serrations, there remains the necessity of an elastic wave that travels at a fraction of the speed of sound and effectively generates the shear band. According to current understanding there is no need for the time scale associated with shear-band initiation to be identical to that of shear-step generation. In fact, a two-stage mechanism has been proposed, which consists of a fast, front-propagating, phonon-controlled initiation phase, generating 
only a small step but causing the structural rejuvenation of the whole band (which was also described as a stress-induced glass transition[24, 27]), followed by a slow, simultaneous shearing phase on the now weakened band that creates the major part of the step (Fig. 2) [6, 9]. The implications of the four different models for event duration $t$, temperature jump $\Delta T$, velocity $v_{\mathrm{SB}}$, and viscosity $\eta$ are summarized in Table 1.

Table 1: Event duration $t$, shear-band velocity $v$, temperature jump $\Delta T$, minimum viscosity $\eta$, and references for the four different models.

\begin{tabular}{cccccc}
\hline \hline & $t(\mathrm{~s})$ & $v(\mathrm{~m} / \mathrm{s})$ & $\Delta T(\mathrm{~K})$ & $\eta(\mathrm{Pa} \mathrm{s})$ & Refs. \\
\hline Model 1 & $10^{-8}$ & $10^{3}$ & 1000 & $10^{-2}$ & {$[12,13]$} \\
Model 2 & $10^{-3}$ & 1 & 90 & 10 & {$[19]$} \\
Model 3 & $10^{-3}$ & $10^{-3}$ & 3 & $10^{4}$ & {$[25]$} \\
Model 4 & $10^{-3}$ & $10^{-3}$ & 3 & $10^{4}$ & {$[6,22]$} \\
\hline \multicolumn{7}{l}{ calculated using $\eta=\sigma_{\mathrm{y}} w /(2 v)$ with $\sigma_{\mathrm{y}} \approx 2 \mathrm{GPa}$ and $w \approx 10 \mathrm{~nm}$}
\end{tabular}

\section{Shear-band temperature}

After this review of shear-step generation models, we will now discuss possible temperature changes within a shear band upon inhomogeneous deformation. A direct assessment of shear-band temperature has so far been impeded by the small size of the shear band and the short time scale of its operation, which both lie significantly below the resolution limits of standard experiments such as IR thermography which are normally used to resolve issues of local heating in materials science [28-30]. Preliminary IR studies of MG deformation [31-33] focused on fatigue and tension, and reported temperature jumps of $3^{\circ} \mathrm{C}$ within regions of shear-band activity. While this method enables measurement of the total amount of released heat, the true shear-band temperature can only be reconstructed by calculations that are highly dependent on the choice of the deformation model (Fig. 1a), as outlined above. Wang et al.[33], for example, assumed instantaneous heating, which led them to estimate a temperature rise within the shear band of about $1200^{\circ} \mathrm{C}$.

In this study, we present the results of high-acquisition rate IR thermography on stable serrated flow in $\mathrm{Zr}_{57.1} \mathrm{Co}_{28.6} \mathrm{Al}_{14.3} \mathrm{MG}$. With this method we have been able to probe an experimental time scale which lies between the time scales postulated by models 1 (phonon time scale) and 4 (serration time scale). Such improved resolution was obtained firstly by using cutting-edge IR technology, and 
secondly by choosing a $\mathrm{Zr}-\mathrm{Co}-\mathrm{Al}$ alloy, which is expected to have particularly slow shear-band dynamics [34].

\section{Experimental procedure}

$\mathrm{Zr}_{57.1} \mathrm{Co}_{28.6} \mathrm{Al}_{14.3}$ metallic glass [35] was produced using an Edmund Bühler arc melter by alloying the high-purity starting materials within a $6 \mathrm{~N}$ Ar atmosphere and subsequent suction casting to rods with a diameter of $2 \mathrm{~mm}$. Coplanar samples with an aspect ratio of 2:1 were produced by grinding and polishing. The samples were equipped with a small notch by Electric Discharge Machining (EDM) [36] in order to initiate the shear band at a free, lateral surface rather than at the contact points of sample and cross-heads. By this means the probability that the shear band will be geometrically arrested shortly after its initiation can be strongly reduced. Compression tests at a rate of $10^{-3} \mathrm{~s}^{-1}$ were carried out using a screw-driven Schenck mechanical testing machine. A $60 \mathrm{kN}$ piezo-cell from Kistler recorded the load signal at a rate of $20 \mathrm{kHz}$, and a strain gauge from Sandner was used to measure the displacement. The compression tests were monitored using a high-speed infrared camera (IRCAM Equus $81 \mathrm{k} \mathrm{SM}$ ) equipped with an InSb detector, which is sensitive to the wavelength interval from 2 to $5 \mu \mathrm{m}$. A fixed frame size of $64 \times 32$ pixels was chosen because it allows a maximum framerate of 3750 frames per second (fps). To ensure precise temperature measurement, the specimens were covered with a thin high-emissivity carbon coating. The temperature measured by the camera was also calibrated using type K thermocouples before testing. Synchronisation of load- and IR signal was established by triggered data acquisition using a customised LabView routine.

\section{Experimental results}

Fig. 3 illustrates the localized heating and subsequent cooling of a line-shaped region, extending from the notch at an angle of $\approx 45^{\circ}$, that appeared in the IR thermographs during stable serrated flow. The frame number for each snapshot (framerate $2500 \mathrm{fps}=0.4 \mathrm{~ms}$ per frame) is indicated. An improvement of contrast was achieved by subtracting a background image, which was obtained by an 
average of the last ten images prior to the onset of heating. Two different sequences of events are observed in Fig. 3. For most of the serrations (ca. 70\%, Fig. 3a), the line-shaped heat source appears within a single frame $(f=1-2)$. Within the course of the next milliseconds, the band widens and the temperature in the centre increases $(f=3-10$, heating rate $260 \mathrm{~K} / \mathrm{s})$. Thereafter, the temperature peaks $\left(f=14, \Delta T \approx 2^{\circ} \mathrm{C}\right)$ and the apparent band starts to cool down slowly, as heat is conducted away towards the anvils. For other serrations (Fig. 3b), the line shape is established via an apparent propagating front over up to 4 subsequent frames $(f=1-4)$. The temperature difference across the front amounts to about $0.15^{\circ} \mathrm{C}$, and the heating rate on the established part of the apparent band is about $50 \mathrm{~K} / \mathrm{s}$. Once it reaches the other side of the sample, the temperature of the apparent band simultaneously increases further along the band $(f=5-10$, heating rate $230 \mathrm{~K} / \mathrm{s})$, until it reaches a peak temperature of $\Delta T \approx 2^{\circ} \mathrm{C}$ $(f=14)$ at a delay of about 10 frames $(=4 \mathrm{~ms})$. Afterwards cooling occurs analogous to the event shown in Fig. 3a. Almost all heat created by an event is conducted away prior to the onset of a subsequent event. In other words, the sample temperature prior to a subsequent heating event is constant. The observed temperature rises involves some degrees Celsius, agreeing well with literature [33].

The sample-spanning line of pixels at which heating was initially observed is assumed to contain the major shear band. By averaging over these pixels, i.e. the apparent shear band, we can quantify the evolution of the apparent shear-band temperature over time. Fig. 4a shows the temporally synchronized signals of stress (black) and apparent shear-band temperature (magenta). The plot is divided into three parts. The first part shows that the first major temperature increase correlates with the first major serration, i.e. with the reaching of peak stress. The few minor events that can be identified before reaching peak stress are attributed to diffuse, non-system spanning events which, we believe, are due to a slight inhomogeneity of the stress state. Apart from the temperature jumps $\Delta T_{\text {jump }}$, a temperature increase during the first serration events causes a background temperature rise $\Delta T_{\text {onset }} \approx$ $0.2^{\circ} \mathrm{C}$, which also remains at larger strains. The middle part of Fig. 4a demonstrates clearly that each serration coincides with one temperature jump. The third part finally gives the remaining flow curve for the sake of completion. The temperature jumps are largest in the intermediate time regime, which is equal to an intermediate strain regime of about $5-15 \%$ because of the constant strain rate. 
Fig. 4b resolves the correlation of a single flow serration with the apparent shear-band temperature during an "instantaneously appearing" event (Fig. 3a). Five stages can be distinguished during the course of a flow serration. Stage (I) represents the end of the elastic loading, i.e. the "stick" part of the serration cycle. In a second stage, (II), stress starts to drop at a moderate rate without inducing a resolvable temperature increase (it is noted that the apparent heat front appearing in the other type of serrations (Fig. 3b) occurs here, causing a minor temperature increase of below $0.2^{\circ} \mathrm{C}$ ). In stage (III), the stress drop rate increases suddenly and coincides with the onset of the temperature jump, which is resolved within at least 8 frames. Reaching the bottom stress level (IV), the drop rate is reduced and transits towards the slow reloading phase $(\mathrm{V})$. On average, temperature jumps $\Delta T_{\text {jump }}$ are on the order of $1.1^{\circ} \mathrm{C}$ and maximum temperature jumps are about $1.9^{\circ} \mathrm{C}$. The onset temperature difference, i.e. the temperature difference at the coordinates of the apparent band prior to a serration event, is about $0.2^{\circ} \mathrm{C}$, which is around $10 \%$ of the peak value.

\section{Discussion}

Shear-band heating is observed to start either simultaneously on the apparent shear plane or, with less probability, to form over a propagating front. It is found that front propagation, which causes only a small temperature increase, coincides with stage II in the stress serration, i.e. with a transient state between the slow stick stage (I) and fast slip phase (III). In serrations of the "instantaneous" type, no temperature rise is monitored in stage II. For both types of serrations, significant heating starts only upon entering stage (III), i.e., the stage at which the AE shear-band initiation signal is also located [24]. We may thus differentiate between some diffuse pre-initiation phase (II) and the pronounced, fast shearing phase (III). In the following, we will focus on the latter.

The fact that the temperature of the apparent major shear band peaks only towards the end of a serration strongly supports the idea that the time scale for temperature rises equals that of the serrations. However, there remains the issue of limited spatial resolution, because we do not directly measure the temperature of the shear band, but the temperature of an apparent band whose thickness is equal to the resolution of the IR thermographs. Naturally, it takes some time for the heat to diffuse 
from the true shear plane over the distance of the method's resolution, which is $w=80 \mu \mathrm{m}$ for our setup. Thus only a comparison between the measured data and the predictions of the different models will allow us to make a strong statement regarding the time scales involved in heating and thus regarding the mode of shear-step formation and shear-band temperature. With model (2) having been discarded (see introductory part) and model (3) having been identified as the result of geometricallyinduced artefacts, we restrict ourselves to comparing the experimental results with the predictions of models (1) and (4), i.e. the phonon-shear-front propagation model and the serration-simultaneous shearing model (4); see Fig. 1a.

In our modelling of shear-band temperature, the true shear band is approximated by an infinitely large plane with zero thickness. According to Ref. [13], the time- and coordinate-dependent temperature jump caused by a constant heat source over an event duration $\Delta t$ is given by

Equation 2

$$
\Delta T_{\text {const }}\left(x, t, q_{s}\right)=\frac{q_{\mathrm{s}}}{2 c_{\mathrm{p}} \alpha}\left(\sqrt{\frac{4 \alpha t}{\pi}} \exp \left(\frac{-x^{2}}{4 \alpha t}\right)-x \operatorname{erfc}\left(\frac{x}{\sqrt{4 \alpha t}}\right)\right)
$$

where $\Delta T$ is the temperature jump, $c_{\mathrm{p}} \approx 3 \times 10^{6} \mathrm{~J} \mathrm{~m}^{-3} \mathrm{~K}^{-1}$ is the specific volume heat capacity, $\alpha \approx 2 \times$ $10^{-6} \mathrm{~m}^{2} \mathrm{~s}^{-1}$ is the thermal diffusivity, $t$ is the time, $x$ is the distance from the heat source and $q_{\mathrm{s}}$ is the heat flux density, which is given as $q_{s}=H / \Delta t$, i.e. the amount of released heat $H$ per area divided by $\Delta t$. The released heat per area is $[12,37]$

Equation 3

$$
H=0.5 \sigma_{\mathrm{y}} \Delta u_{\mathrm{pl}}
$$

where $\sigma_{\mathrm{y}}$ is the yield strength.

In the case of model (1), the time scale of observation (milliseconds) is much greater than the time scale of assumed event duration (nano- to microseconds). Therefore, the time-dependent temperature jumps at the time scale of observation can be approximated well by instantaneous heating [12]:

Equation 4

$$
\Delta T_{\text {inst }}(x, t)=\left(\frac{H}{2 c_{\mathrm{p}} \sqrt{\pi \alpha}}\right) \frac{1}{\sqrt{t}} \exp \left(\frac{-x^{2}}{4 \alpha t}\right)
$$


For model (4), with an input time scale on the same order of magnitude as the time scale of observation $\Delta t$, Equation 2 is used as a direct starting point which we will refer to as model (4a). This model has two limitations, however, which we account for in the following to obtain an improved model (4b). First, Equation 2 is only valid as long as the heating source is active. It can therefore not be used to model temperature evolution for times longer than $\Delta t$. This limitation can be overcome by calculating the temperature evolution once the heating source is switched off, which gives

Equation 5

$$
\Delta T_{\mathrm{off}}\left(x, t_{\mathrm{off}}, H_{i}\right)=\int_{\xi=0}^{d} \Delta T_{\mathrm{inst}}\left(x-\xi, t_{\mathrm{off}}, H_{i}\left(x-\xi, \Delta t, q_{s}\right)\right) d \xi
$$

where $\xi$ is a space variable, $d$ is the distance at which the temperature rise becomes insignificant (in our case $d=500 \mu \mathrm{m}), t_{\text {off }}$ is the time elapsed since the source was switched off, and $H_{i}\left(x, t_{0}, q_{s}\right)=\int_{\xi}^{\xi+\Delta \xi} \Delta T_{\text {const }}\left(x, \Delta t, q_{s}\right) c_{\mathrm{p}} d \xi$, i.e. the heat content just prior to switch-off, is discretized in spaces $\Delta \xi$. In other words, the thermal profile just before switch-off is converted into a discrete heat profile, and the components are then treated as instantaneous heat sources according to Equation 4, which, added together, will provide the total temperature at a given coordinate and time. The second limitation of Equation 2 is that constant heating rate has the tendency to oversimplify the problem, as acknowledged in Ref. [13]. Having recorded stress drops with high acquisition rates, we are, however, also able to overcome this limitation and to directly calculate a time-dependent heating rate from the derivative of the stress signal during a serration. This is achieved by combining Equation 1 and Equation 3, which gives

Equation 6

$$
q_{\mathrm{S}}(t)=\frac{1}{2} \sigma(t) \frac{d \sigma}{d t} A\left(C_{\mathrm{M}}+\mathrm{C}_{\mathrm{S}}\right)
$$

where $A$ is the sample cross-section, and the yield stress $\sigma_{\mathrm{y}}$ in Eq. 3 has been substituted by the timedependent stress during a serration, $\sigma(t)$. Equation 6 allows us to predict a temperature evolution proportional to the decrease in potential energy of the machine-sample assembly. The time- and spacedependent temperature jump for model (4b), which we call "proportional heating", thus becomes 


$$
\Delta T_{\text {prop }}(x, t)=\int_{\tau=0}^{t} \Delta T_{\text {off }}\left(x, \tau-\Delta \tau, H\left(x, \Delta \tau, q_{\mathrm{s}}(\tau)\right)\right) d \tau
$$

where $\Delta \tau$ is the discrete time step of the numerical integration. Equation 7 thus represents the sum of all contributions to the total temperature jump caused by the heat sources $q_{\mathrm{s}}$ that were active for durations $\Delta \tau$ at times $\tau \leq t$, each of which can be calculated with Equation 5, i.e. with the abovementioned switched-off source method.

Models (1), (4a), and (4b) are now tested during stages III and IV of the serration displayed in Fig. 5a. The time-dependent heating rate per area, i.e. heat flux density $q_{\mathrm{s}}$ (Equation 6), is shown in Fig. 5b. For the calculations we used an effective heating rate which is set to zero except for serration stages III and IV. The time at which stage III starts will also be used as a time reference for the positioning of the curve obtained for models (1) and (4a).

Fig. 5c shows how the experimentally determined shear-band temperature compares with the various model predictions evaluated at $x=80 \mu \mathrm{m}$. Shear-step generation according to model (1) (magenta) generates a rapid temperature increase upon shear-band initiation when entering stage III. Here, the maximum of the temperature jump $\left(2.6^{\circ} \mathrm{C}\right)$ is reached within $1.6 \mathrm{~ms}$. Thus model (1) not only overestimates the temperature jump but also the time-dependent heating rate (slope). Converse behaviour is observed for shear-band heating at a constant rate (model (4a), cyan), where the heating rate is underestimated at the beginning of the serration and slightly overestimated towards the end of the event. Thus, while model (4a) provides realistic values for the temperature jump it fails to match the time-dependent heating of the apparent band. Clearly, model (4b), which describes heating to be proportional to the elastic-energy release, matches the experimental data best. Both the time-dependent heating rate (slope) and the peak temperature are reproduced very efficiently by the simulation. This is observed throughout the set of 80 serrations. Having now accounted for thermal diffusion from the shear plane to the apparent band, which has the thickness of one pixel, we can thus conclude that the temperature trace observed cannot be caused by the process described by model (1). Instead, Fig. 5 provides strong evidence that the shear step is mostly caused by a process of simultaneous shear (Fig. 1a, model 4), which occurs at the time scales obtained by high-acquisition rate instrumented mechanical testing. 
Fig. 6 now addresses the emerging question of true shear-band temperature. To this end, Fig. 6a shows the histograms of the experimentally recorded temperature jumps. The apparent band temperatures simulated by models (1) and (4b) are shown by the open columns in Fig. $6 \mathrm{~b}$ and c, whereas the solid columns in these figures refer to the temperatures obtained when the models are evaluated at $x=0$, i.e. right at the position of the shear plane. In the current calculation, Equation 2 with $t=\Delta t=10^{-8} \mathrm{~s}$ was used for model (1). While model (1) predicts shear-band temperature rises of 2000 to $4000^{\circ} \mathrm{C}$, model (4b) predicts only moderate rises of $2-6^{\circ} \mathrm{C}$ for the actual shear plane. Because model (4b) was found to agree best with the experimental data in terms of the heating time of the apparent band (Fig. 5c), we can clearly conclude that temperature rises during shear banding are very moderate. These results may conclude the long-standing debate about the dynamics of shear banding and the resulting temperature increase.

\section{Conclusions}

In summary, we have reviewed the current models of shear-step generation during inhomogeneous deformation and carried out time-resolved shear-band heating experiments on $\mathrm{Zr}-\mathrm{Co}-$ Al MGs during compressive stable, serrated flow. We measured maximum temperature jumps of 1$2^{\circ} \mathrm{C}$ for an apparent band of $80 \mu \mathrm{m}$ thickness and were able to show clearly that each temperature rise coincides with the end of the slip phase in a flow serration. Accounting for thermal diffusion and comparing the experimental results with two of the reviewed deformation models, namely shear-band heating at time scales of elastic wave propagation (model (1): phonon-shear-front propagation) and shear-band heating at time scales of flow serrations (model (4): serration-simultaneous shear), we find strong evidence for the validity of model (4). Using this model, our results then provide evidence that shear-band heating in metallic glasses is less than $2-6^{\circ} \mathrm{C}$ during stable serrated flow. More pronounced shear-band temperature jumps may be expected for larger samples with higher stress levels and increased shear-band dynamics, as can be concluded from an evaluation of Equation 6. In general, however, model (4) adequately describes shear-band dynamics in metallic glasses, and temperature 
rises during shear banding are very moderate. Our findings may conclude a long-standing debate in this area of research.

\section{Acknowledgments}

The authors gratefully acknowledge funding by the Swiss National Science Foundation (SNF Grant No. 200020-153103). They also thank Josef Hecht for setting up the LabView routine for synchronized recording of stress and IR camera signals.

\section{References}

[1] T. Masumoto, R. Maddin, The mechanical properties of palladium 20 a/o silicon alloy quenched from the liquid state, Acta Metall. 19 (1971) 725-741.

[2] H. Leamy, T. Wang, H. Chen, Plastic flow and fracture of metallic glass, Metall. Mater. Trans. B 3 (1972) 699-708.

[3] C.A. Pampillo, Localized shear deformation in a glassy metal, Scr. Met. Mater. 6 (1972) 915917.

[4] C.A. Schuh, T.C. Hufnagel, U. Ramamurty, Mechanical behavior of amorphous alloys, Acta Mater. 55 (2007) 4067-4109.

[5] A.L. Greer, Y.Q. Cheng, E. Ma, Shear bands in metallic glasses, Mater. Sci. Eng. R 74 (2013) $71-$ 132.

[6] R. Maaß, J.F. Löffler, Shear-Band Dynamics in Metallic Glasses, Adv. Funct. Mater. 25 (2015) 2353-2368.

[7] Y. Zhang, A.L. Greer, Thickness of shear bands in metallic glasses, Appl. Phys. Lett. 89 (2006) 071907.

[8] H. Neuhäuser, Rate of shear band formation in metallic glasses, Scr. Met. Mater. 12 (1978) 471-474.

[9] D. Klaumünzer, R. Maaß, J.F. Löffler, Stick-slip dynamics and recent insights into shear banding in metallic glasses, J. Mater. Res. 26 (2011) 1453-1463.

[10] H. Chen, T. Wang, Mechanical Properties of Metallic Glasses of Pd-Si-Based Alloys, J. Appl. Phys. 41 (1970) 5338.

[11] Y.Q. Cheng, Z. Han, Y. Li, E. Ma, Cold versus hot shear banding in bulk metallic glass, Phys. Rev. B 80 (2009) 134115.

[12] D.B. Miracle, A. Concustell, Y. Zhang, A.R. Yavari, A.L. Greer, Shear bands in metallic glasses: Size effects on thermal profiles, Acta Mater. 59 (2011) 2831-2840.

[13] K. Georgarakis, M. Aljerf, Y. Li, A. LeMoulec, F. Charlot, A.R. Yavari, K. Chornokhvostenko, E. Tabachnikova, G.A. Evangelakis, D.B. Miracle, A.L. Greer, T. Zhang, Shear band melting and serrated flow in metallic glasses, Appl. Phys. Lett. 93 (2008) 031907.

[14] H. Guo, J. Wen, N.M. Xiao, Z.F. Zhang, M.L. Sui, The more shearing, the thicker shear band and heat-affected zone in bulk metallic glass, J. Mater. Res. 23 (2008) 2133-2138.

[15] J.J. Lewandowski, A.L. Greer, Temperature rise at shear bands in metallic glasses, Nat. Mater. 5 (2006) 15-18. 
[16] S.K. Slaughter, F. Kertis, E. Deda, X. Gu, W.J. Wright, T.C. Hufnagel, Shear bands in metallic glasses are not necessarily hot, APL Materials 2 (2014) 096110.

[17] S.X. Song, H. Bei, J. Wadsworth, T.G. Nieh, Flow serration in a Zr-based bulk metallic glass in compression at low strain rates, Intermetallics 16 (2008) 813-818.

[18] R. Maaß, D. Klaumünzer, E.I. Preiß, P.M. Derlet, J.F. Löffler, Single shear-band plasticity in a bulk metallic glass at cryogenic temperatures, Scr. Mater. 66 (2012) 231-234.

[19] W.J. Wright, R. Saha, W.D. Nix, Deformation mechanisms of the $\mathrm{Zr}_{40} \mathrm{Ti}_{14} \mathrm{Ni}_{10} \mathrm{Cu}_{12} \mathrm{Be}_{24}$ bulk metallic glass, Mater. Trans. 42 (2001) 642-649.

[20] R. Maaß, D. Klaumünzer, J.F. Löffler, Propagation dynamics of individual shear bands during inhomogeneous flow in a Zr-based bulk metallic glass, Acta Mater. 59 (2011) 3205-3213.

[21] D. Klaumünzer, R. Maaß, F.H. Dalla Torre, J.F. Löffler, Temperature-dependent shear band dynamics in a Zr-based bulk metallic glass, Appl. Phys. Lett. 96 (2010) 061901.

[22] S.X. Song, X.L. Wang, T.G. Nieh, Capturing shear band propagation in a Zr-based metallic glass using a high-speed camera, Scr. Mater. 62 (2010) 847-850.

[23] W.J. Wright, M.W. Samale, T.C. Hufnagel, M.M. LeBlanc, J.N. Florando, Studies of shear band velocity using spatially and temporally resolved measurements of strain during quasistatic compression of a bulk metallic glass, Acta Mater. 57 (2009) 4639-4648.

[24] D. Klaumünzer, A. Lazarev, R. Maaß, F.H. Dalla Torre, A. Vinogradov, J.F. Löffler, Probing Shear-Band Initiation in Metallic Glasses, Physical Review Letters 107 (2011) 185502.

[25] S.-H. Joo, H. Kato, K. Gangwar, S. Lee, H.S. Kim, Shear banding behavior and fracture mechanisms of $\mathrm{Zr}_{55} \mathrm{Al}_{10} \mathrm{Ni}_{5} \mathrm{Cu}_{30}$ bulk metallic glass in uniaxial compression analyzed using a digital image correlation method, Intermetallics 32 (2013) 21-29.

[26] R.T. Qu, Z.Q. Liu, G. Wang, Z.F. Zhang, Progressive shear band propagation in metallic glasses under compression, Acta Mater. 91 (2015) 19-33.

[27] P. Guan, M. Chen, T. Egami, Stress-Temperature Scaling for Steady-State Flow in Metallic Glasses, Phys. Rev. Lett. 104 (2010) 205701.

[28] H. Ait-Amokhtar, C. Fressengeas, S. Boudrahem, The dynamics of Portevin-Le Chatelier bands in an Al-Mg alloy from infrared thermography, Mater. Sci. Eng. A 488 (2008) 540-546.

[29] M.P. Luong, Fatigue limit evaluation of metals using an infrared thermographic technique, Mechanics of Materials 28 (1998) 155-163.

[30] O. Plekhov, T. Palin-Luc, N. Saintier, S. Uvarov, O. Naimark, Fatigue crack initiation and growth in a 35CrMo4 steel investigated by infrared thermography, Fatigue Fract. Eng. M. 28 (2005) 169-178.

[31] J.J. Luo, G.Y. Wang, H.R. Qi, Y. Yokoyama, P.K. Liaw, A. Inoue, Interpreting temperature evolution of a bulk-metallic glass during cyclic loading through spatial-temporal modeling, Intermetallics 29 (2012) 1-13.

[32] B. Yang, P.K. Liaw, M. Morrison, C.T. Liu, R.A. Buchanan, J.Y. Huang, R.C. Kuo, J.G. Huang, D.E. Fielden, Temperature evolution during fatigue damage, Intermetallics 13 (2005) 419-428.

[33] G. Wang, Q.M. Feng, B. Yang, W.H. Jiang, P.K. Liaw, C.T. Liu, Thermographic studies of temperature evolutions in bulk metallic glasses: An overview, Intermetallics 30 (2012) 1-11.

[34] P. Thurnheer, R. Maaß, K.J. Laws, J.F. Löffler, Activation energies determining shear stability in metallic glasses, (unpublished) (2016).

[35] J.B. Qiang, X.Z. Ding, Y.M. Wang, Q. Wang, Q.M. Duan, C. Dong, A Zr-Co-Al Bulk Metallic Glass Derived from the Atomic-Cluster-Plus-Glue-Atom Model and Its Mechanical Properties, Transactions of the Indian Institute of Metals 65 (2012) 577-580.

[36] R. Maaß, D. Klaumünzer, G. Villard, P.M. Derlet, J.F. Löffler, Shear-band arrest and stress overshoots during inhomogeneous flow in a metallic glass, Appl. Phys. Lett. 100 (2012) 071904.

[37] Y. Zhang, N.A. Stelmashenko, Z.H. Barber, W.H. Wang, J.J. Lewandowski, A.L. Greer, Local temperature rises during mechanical testing of metallic glasses, J. Mater. Res. 22 (2007) 419-427. 

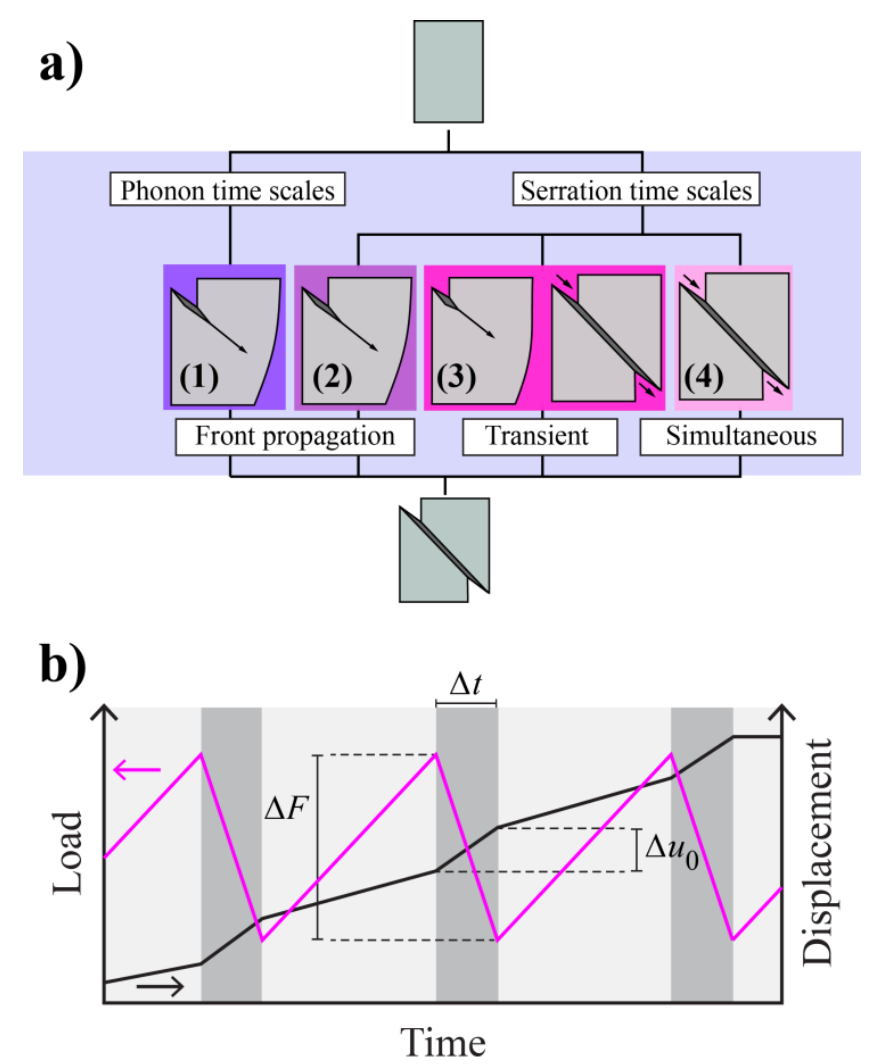

Fig. 1. a) Four scenarios of shear-step generation in MGs: (1) phonon-shear-front propagation, (2) serration-shear-front propagation, (3) serration-transient behaviour, and (4) serration-simultaneous shear. b) Schematic of the load- and displacement-signal of three serrations during MG plastic deformation. The serration parameters load drop $\Delta F$, event duration $\Delta t$, and apparent displacement jump $\Delta u_{0}$ are indicated. Slow "stick" phases are shaded in light grey and fast "slip" phases in darker grey.

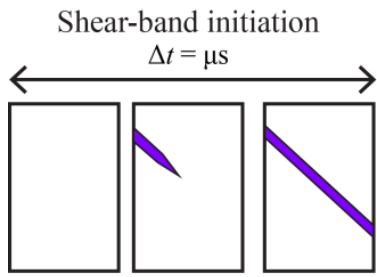

a) b) c)

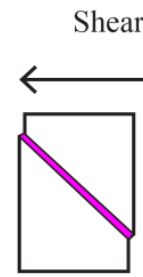

d)
Shear-band propagation $\Delta t=\mathrm{ms}$

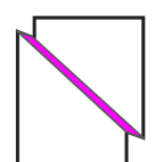

e)

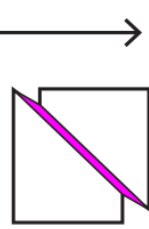

f)

Fig. 2. Idealized shear-band dynamics. A shear band is initiated within microseconds by a front-dilating shear wave (a-c). In this stage, only minor amounts of plastic deformation are accumulated. In the subsequent propagation phase (d-f), macroscopic plasticity is obtained within the shear band via simultaneous shear occurring within milliseconds. 

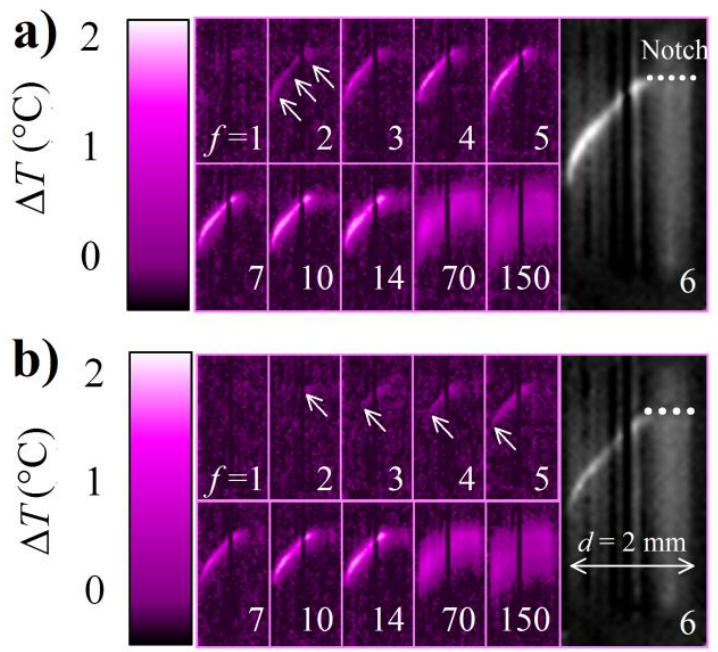

Fig. 3. Localized heating during serrated flow. a) Instantaneous appearance of the shear band, and b) propagating heat front; $f$ indicates the frame number, whereby 1 frame is measured within $0.4 \mathrm{~ms}$.
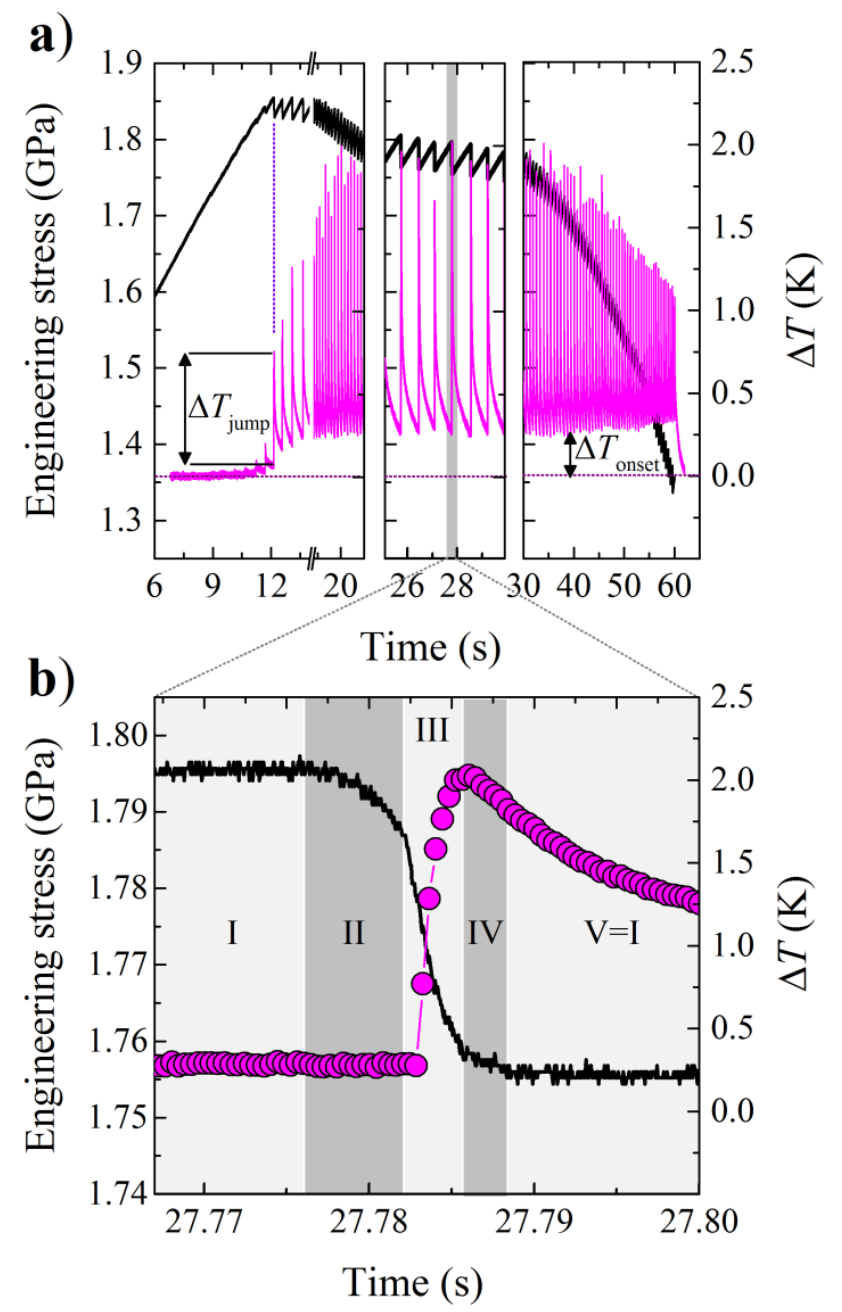

Fig. 4. Serrated flow curve and temperature of the apparent shear band as a function of time. a) Throughout the experiment, each temperature jump corresponds to a single serration. b) Close-up of an individual serration. In the stress signal, the different stages (I) slow loading, (II) transition to unloading, (III) fast unloading, (IV) arrest and (V) reloading (= I) are indicated. A significant temperature rise is only observed in stage III. 
Time (ms)

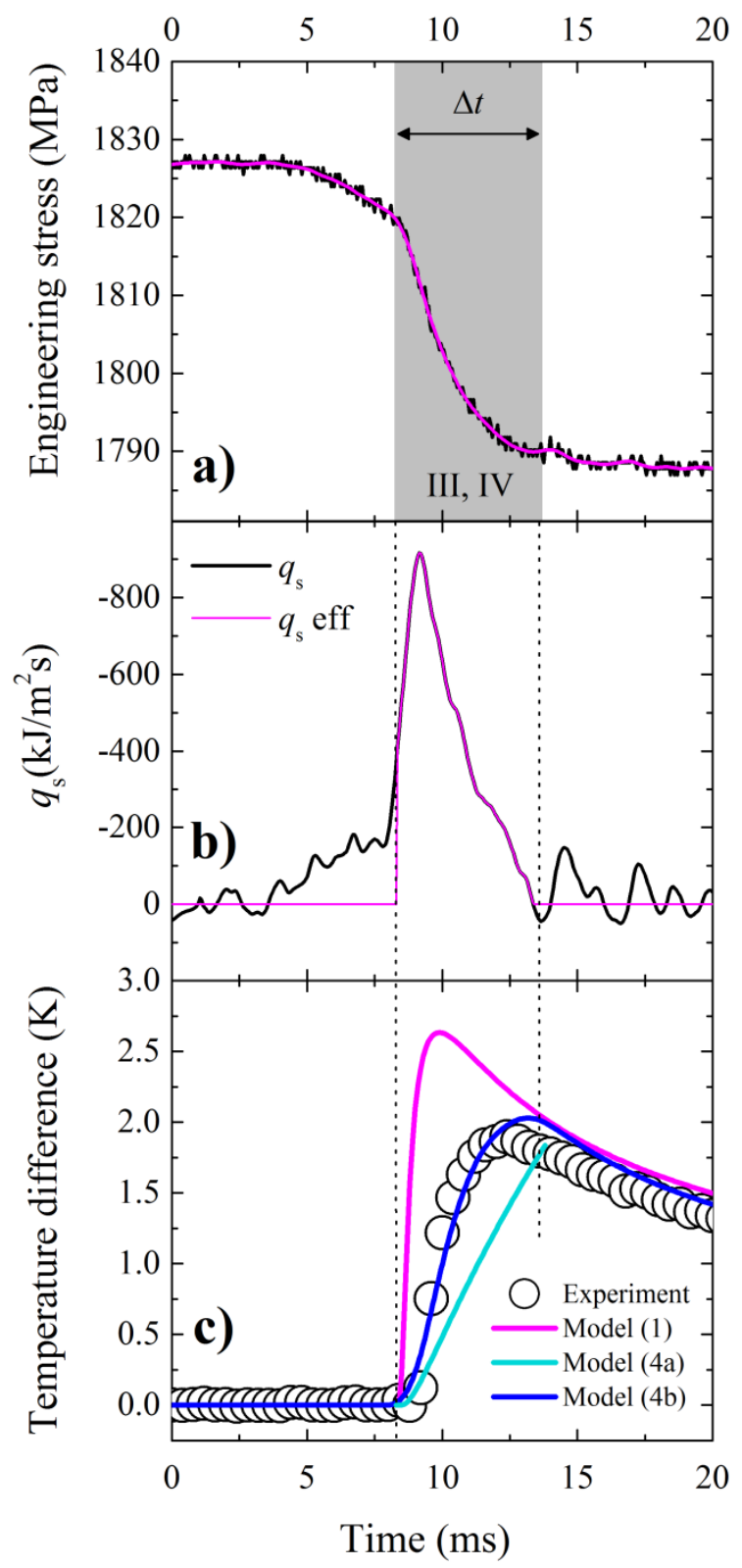

Fig. 5. a) Raw and smoothened stress-drop data. b) Raw and effective heating rate per area (heat flux density) as a function of time. c) Comparison of the experimental temperature trace (circles) with the predictions of model (1) (phonon-shear-front propagation, magenta), model (4a) (serration-simultaneous shear with a constant heating rate, cyan), and model (4b) (serration-simultaneous shear with a heating rate proportional to the elastic-energy release, blue). 


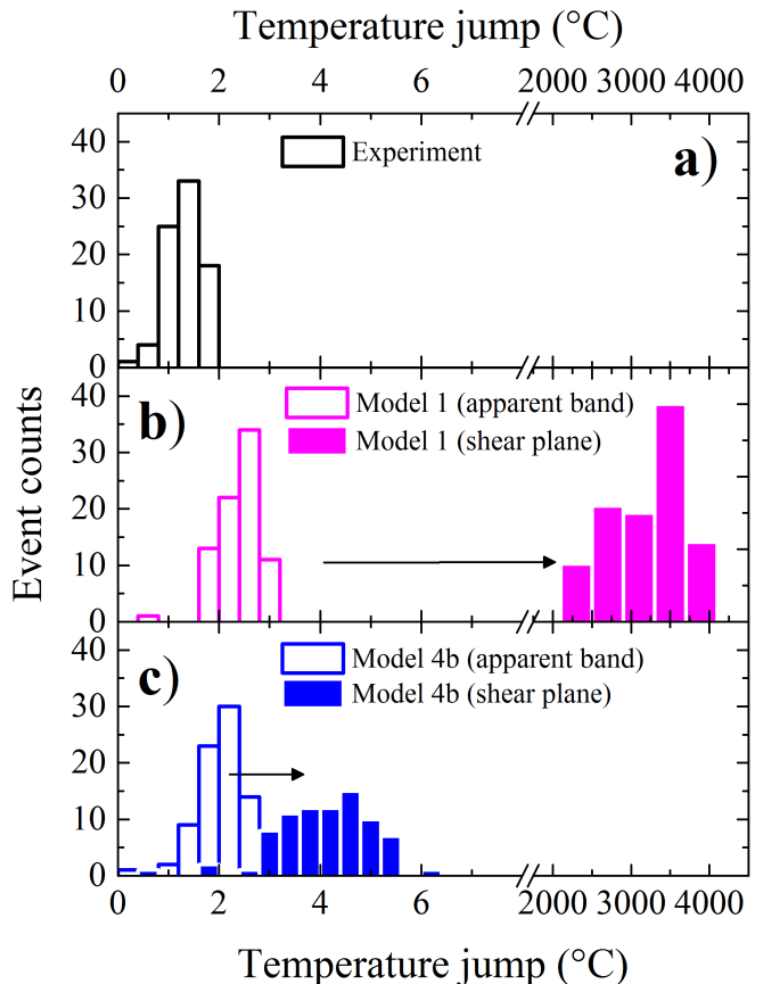

Fig. 6. Histograms of shear-band temperatures. (a) Experimentally measured temperature jumps of the apparent shear band (which has the size of a pixel); (b) temperature jumps simulated by model (1) for the apparent band (open columns) and for the shear plane (solid columns); and (c) temperature jumps simulated by model (4b) for the apparent band (open columns) and for the shear plane (solid columns). 